\title{
Semen profiles of young men involved as bicycle taxi cyclists in Mangochi District, Malawi: A case-control study
}

W Kipandula ${ }^{1}$, F Lampiao ${ }^{2}$

1. Department of Medical Laboratory Sciences, College of Medicine, University of Malawi, Blantyre, Malawi

2. Department of Basic Medical Sciences, Division of Physiology, College of Medicine, University of Malawi, Blantyre, Malawi.

Corresponding author: Fanuel Lampiao, Department of Basic Medical Sciences, Division of Physiology, College of Medicine, P.O. Box 360, Blantyre 3, Malawi. Phone: +265 01878 058, Fax: +265 01872644 , Email: flampiao@medcol.mw

\section{Abstract}

\section{Aim}

The purpose of this study was to evaluate the semen profiles of bicycle taxi cyclists and healthy controls in Mangochi district, Malawi.

\section{Methods}

Semen samples were collected from young bicycle taxi cyclists after two to three days of sexual abstinence. A control group, comprising young men who were not bicycle taxi operators also submitted semen samples. Samples were left to liquefy for 30 minutes before measurements were conducted of volume, concentration, total motility, and progressive motility. This was followed by preparation of morphology slides. Light microscopy was used for sperm analysis.

\section{Results}

Semen parameters such as volume $(1.66 \pm 0.18 \mathrm{~mL}$ vs. $3.64 \pm 0.17 \mathrm{~mL}$; $\mathrm{p}$ $=0.0001)$, concentration $(28.31 \pm 4.33 \times 106 / \mathrm{mL}$ vs. $54.95 \pm 5.93 \times 106 /$ $\mathrm{mL} ; \mathrm{p}=0.02)$, total motility $(56.98 \% \pm 8.22 \%$ vs. $56.98 \% \pm 8.22 \% ; \mathrm{p}$ $=0.03)$, progressive motility $(22.57 \% \pm 3.35 \%$ vs. $59.69 \% \pm 4.82 \% ; p$ $=0.004)$, and morphology $(6.98 \% \pm 3.23 \%$ vs. $19.73 \% \pm 2.32 \% ; \mathrm{p}=$ $0.006)$ were significantly reduced in the bicycle taxi cyclists compared to the healthy controls.

\section{Conclusion}

In this case-control study, bicycle taxi operators had lower semen volume, concentration, total motility, and progressive motility, as well as a higher concentration of abnormally shaped spermatocytes, compared to healthy controls.

\section{Introduction}

Male reproductive function seems to have deteriorated considerably during the past four to five decades among healthy young men in the general population ${ }^{1}$. In recent years, infertility has become an increasingly important aspect of health services ${ }^{1}$. Poor semen quality has been suggested as possibly contributing to reduced fertility ${ }^{2}$. It has been observed that there is a significant decline in mean sperm concentration from $113 \times 106 / \mathrm{ml}$ in 1940 to $66 \times 106 / \mathrm{ml}$ in the $1990 \mathrm{~s}^{3,4}$

Male factor infertility is responsible for $30 \%$ of infertility cases, whereas $30 \%$ are attributed to female factors. An additional $30 \%$ of cases have a contribution of factors from both partners, and $10 \%$ are idiopathic ${ }^{5}$. Certain cases of male infertility are related to anatomical and chromosomal abnormalities, but an estimated $40 \%$ to $90 \%$ are associated with deficient sperm production of unidentifiable origin or a combination of various factors ${ }^{3}$. These factors could be related to lifestyle, occupational, or other environmental influences. For example, obesity has been suggested a risk factor for poor semen quality ${ }^{6}$.
Cycling has been linked to genitourinary problems, ${ }^{7,8}$ including nerve entrapment syndromes (50\% to $91 \%$ of cyclists), erectile dysfunction (13\% to $24 \%$ ), and other less common symptoms (priapism, penile thrombosis, haematuria, torsion of the spermatic cord, perineal nodular induration, and prostatitis $)^{8}$. It has been reported that cycling more than five hours a week is associated with low sperm concentration? A high inverse correlation between sperm morphology (percentage of normal forms) and the weekly volume of cycling in triathletes has also been reported ${ }^{10}$. Additionally, it is well known that sperm production is sensitive to high body temperatures, ${ }^{3}$ and this might contribute to the effect that strenuous cycling has on semen quality.

In rural Malawi there has been an increase in the use of bicycle taxis as a means of transportation in recent years. Mangochi is one of the districts in the country that has experienced this rise in bicycle taxi use. Bicycle taxi operators earn a living by transporting people from one place to another from morning to evening every day. There is no specific report on the association of bicycle taxi operation and reduced fertility or semen parameters, and we speculated that bicycle taxi cyclists in Malawi may have poor sperm quality, given their strenuous daily cycling routines. Thus, the aim of the present study was to examine the association between the cycling and the quality of sperm among bicycle taxi operators.

\section{Materials and Methods}

This study was conducted between October and November 2014. A total of 36 young men aged 18 to 40 years who had been bicycle taxi cyclists for not less than two years in Mangochi District were recruited. The control group consisted of 20 normozoospermic healthy volunteers aged 18 to 40 years who were not bicycle taxi cyclists. All participants provided semen samples after providing informed consent. The study was conducted in accordance with the Declaration of Helsinki and was approved by the University of Malawi's College of Medicine Research and Ethics Committee (COMREC).

\section{Inclusion and exclusion criteria}

Men aged between 18 and 40 years who were not known to have impaired reproductive function were eligible. Those excluded were men younger than 18 years and older than 40 years. We also excluded those who were overweight or obese (body mass index, BMI $>25 \mathrm{~kg} / \mathrm{m} 2$ ), diabetics, and smokers.

\section{Semen sample collection and processing}

Semen samples were collected from participants, via masturbation, into sterile containers after two to three days of sexual abstinence. Semen assessment was carried out in accordance to the method described by the World Health Organization (WHO), ${ }^{3}$ as soon as the samples were liquefied, within one hour from collection. A graduated pipette, accurate to within $0.1 \mathrm{~mL}$, was used to measure seminal volume. After appropriate dilution, a haemo-cytometer (improved Neubauer counting chamber) was used to determine sperm concentration. Sperm total motility and progressive motility were assessed by direct observation under a microscope (400x). Smears were made on clean slides, air dried after, http://dx.doi.org/10.4314/mmj.v27i4.7 
and then stained with Hemacolor (Merck, Darmstadt, Germany). Morphology was assessed under oil immersion light microscopy according to the Tygerberg strict criteria.

\section{Statistical analysis}

Data were analyzed using the Prism 4 statistical programme (GraphPad, San Diego, CA, USA). All data are expressed as mean \pm SEM. Student's t-test and one-way ANOVA (with Bonferroni post hoc test if $\mathrm{p}<0.05$ ) were used for statistical analysis. Differences were regarded statistically significant if $\mathrm{p}<0.05$.

\section{Results}

\section{Participant characteristics}

The mean age for the bicycle taxi cyclists was $24 \pm 0.8$ years, compared to $22 \pm 0.7$ years for the non-cyclist controls (Table 1); this difference was not statistically significant.

\section{Table 1: Characteristics of Cyclists and Non-Cyclists}

\begin{tabular}{|lll|}
\hline Characteristic & Non-cyclists & Cyclists \\
\hline Age (years) & $22 \pm 0.7$ & $24 \pm 0.8$ \\
Number of participants & 20 & 36 \\
Number of years cycled & 0 & 2.7 \\
Body mass index $\left(\mathrm{kg} / \mathrm{m}^{2}\right)$ & $21.71 \pm 2.45$ & $20.65 \pm 1.92$ \\
\hline
\end{tabular}

\section{Semen analysis}

This study found that all the measured semen parameters (volume, concentration, total motility, progressive motility, percent normal morphology) were significantly reduced in the cyclist group compared to the non-cyclist group ( $\mathrm{p}<$ 0.05 ), as indicated in Table 2. As shown in Table 3, there was a general downward trend for all measured semen parameters as the number of years of operating a bicycle taxi increased.

\section{Tables 283: Semen Parameters of Cyclists and Non-Cyclists}

\begin{tabular}{|llll|}
\hline \hline Semen parameter & Non-cyclists & Cyclists & p-value \\
\hline Volume $(\mathrm{mL})$ & $3.64 \pm 0.17$ & $1.66 \pm 0.18$ & 0.0001 \\
Concentration $\left(10^{6} / \mathrm{mL}\right)$ & $54.95 \pm 5.93$ & $28.31 \pm 4.33$ & 0.02 \\
Total motility & $83.43 \% \pm 6.97 \%$ & $56.98 \% \pm 8.22 \%$ & 0.03 \\
Progressive motility & $59.69 \% \pm 4.82 \%$ & $22.57 \% \pm 3.35 \%$ & 0.004 \\
Normal morphology & $19.73 \% \pm 2.32 \%$ & $6.98 \% \pm 3.23 \%$ & 0.006 \\
\hline
\end{tabular}

\begin{tabular}{|lllll|}
\hline & \multicolumn{4}{c|}{ Years cycled } \\
\cline { 2 - 5 } Semen parameter & Non-cyclists & $\mathbf{2 - 3}$ years & 4-5 years & $>5$ years \\
\hline Volume $(\mathrm{mL})$ & $3.64 \pm 0.17$ & $1.53 \pm 0.17^{*}$ & $1.88 \pm 0.21^{*}$ & $1.43 \pm 0.39^{*}$ \\
Concentration $\left(10^{6} / \mathrm{mL}\right)$ & $54.95 \pm 5.93$ & $27.81 \pm 5.12^{*}$ & $22.06 \pm 5.21^{*}$ & $19.85 \pm 4.46^{*}$ \\
Total motility (\%) & $83.43 \pm 6.97$ & $58.45 \pm 7.98^{*}$ & $55.34 \pm 6.96^{*}$ & $53.23 \pm 7.02^{*}$ \\
Progressive motility (\%) & $59.69 \pm 4.82$ & $22.64 \pm 4.26^{*}$ & $20.14 \pm 6.18^{*}$ & $16.33 \pm 3.11^{*}$ \\
Normal morphology (\%) & $19.73 \pm 2.32$ & $8.66 \pm 2.34^{*}$ & $7.63 \pm 2.11^{*}$ & $7.21 \pm 2.43^{*}$ \\
\hline *p $<0.05$ (compared to non-cyclists) & & & & \\
& & & & \\
\hline
\end{tabular}

\section{Discussion}

The findings of this study showed that operating a bicycle taxi reduced semen quality, as indicated by the lower semen volume, concentration, total motility, progressive motility, and percent normal morphology in the cycling group. It was also observed in this study that the longer one is involved in the bicycle taxi business, the poorer his semen quality. These results suggest that excessive cycling, while carrying a heavy load on the bicycle, negatively affects semen parameters. These findings agree with earlier studies that have shown deleterious effects of bicycling on semen parameters among competitive cyclists ${ }^{11,12}$. It has been reported that cycling is associated with increased scrotal temperatures with time and, consequently, with impaired semen quality ${ }^{13}$.

A few studies that have evaluated the relationship between bicycling and semen quality have been confined to assess one or two semen parameters ${ }^{11,12}$. This study has assessed a number of semen parameters that function as indicators of male fertility, status such as volume, concentration, total motility, progressive motility, and percent normal morphology. The semen parameters that were most altered by bicycling were volume, progressive motility, and percent normal morphology. The reduction in semen volume suggests insufficiency of the reproductive accessory glands, such as the prostate, seminal vesicles, and bulbourethral glands ${ }^{13}$. It is therefore imperative for future studies to assess testicular and prostate disorders in these bicycle taxi operators. Other factors, such as reactive oxygen species, which are well known to adversely influence seminal plasma and spermatozoa, must also be thoroughly assessed so that ways of preventing their generation or mitigating their effects (thorough antioxidant supplementation, for example) can be explored $^{14,15}$. BMIs were not significantly different between the two groups, thus we could not attribute the differences in semen parameters to BMI.

This study did not assess the hormonal profiles of the participants, which can be considered a limitation. However, analyzing hormonal profiles would have likely contributed limited additional insight, because semen alterations often occur without associated hormonal abnormalities. In fact, the complex of the physiologic mechanisms involved in regulating levels of follicle stimulating hormone, luteinizing hormone, inhibin $\mathrm{B}$, testosterone, and thyroid hormones are not yet understood well enough to know if these hormones indeed have a predictive value on semen quality ${ }^{16,17}$.

This study recommends that bicycle taxi operators must be informed about how cycling may negatively affect their reproductive health, as these are usually young men of reproductive age.

\section{Funding}

This study was funded by the office of the Postgraduate Dean, College of Medicine, University of Malawi

\section{References}

1. Andersen AG, Jensen TK, Carlsen E, Jørgensen N, Andersson AM, Krarup T, Keiding N, Skakkebaek NE. High frequency of sub-optimal semen quality in an unselected population of young men. Hum Reprod. $2000 \mathrm{Feb} ; 15(2): 366-72$.

2. Auger J, Kunstmann JM, Czyglik F, Jouannet P. Decline in semen quality among fertile men in Paris during the past 20 years. N Engl J Med. 1995 Feb 2;332(5):281-5. 
3. World Health Organization. WHO laboratory manual for the examination of human semen and sperm-cervical mucus interaction. 4th ed. Cambridge: Cambridge University Press; 2010.

4. Carlsen E, Giwercman A, Keiding N, Skakkebaek NE. Evidence for decreasing quality of semen during past 50 years. BMJ. 1992 Sep 12;305(6854):609-13.

5. Irvine S, Cawood E, Richardson D, MacDonald E, Aitken J. Evidence of deteriorating semen quality in the United Kingdom: birth cohort study in 577 men in Scotland over 11 years. BMJ. 1996 Feb 24;312(7029):467-71.

6. Hammoud AO1, Wilde N, Gibson M, Parks A, Carrell DT, Meikle AW. Fertil Steril. 2008 Dec;90(6):2222-5. doi: 10.1016/j. fertnstert.2007.10.011.

7. Asplund C, Barkdull T, Weiss BD. Genitourinary problems in bicyclists. Curr Sports Med Rep. 2007 Oct;6(5):333-9.

8. Leibovitch I, Mor Y. The vicious cycling: bicycling related urogenital disorders. Eur Urol. 2005 Mar;47(3):277-86; discussion 286-7.

9. Wise LA, Cramer DW, Hornstein MD, Ashby RK, Missmer SA. Physical activity and semen quality among men attending an infertility clinic. Fertil Steril. 2011 Mar 1;95(3):1025-30. doi: 10.1016/j. fertnstert.2010.11.006.

10. Vaamonde D, Da Silva-Grigolettob ME, García-Manso JM, Cunha-Filho JS, Vaamonde-Lemos R. Sperm morphology normalcy is inversely correlated to cycling kilometers in elite triathletes . Rev Andal Med Deporte. 2009;2(2):43-6.
11. Lucía A, Chicharro JL, Pérez M, Serratosa L, Bandrés F, Legido JC. Reproductive function in male endurance athletes: sperm analysis and hormonal profile. J Appl Physiol (1985). 1996 Dec;81(6):2627-36.

12. Gebreegziabher Y, Marcos E, McKinon W, Rogers G. Sperm characteristics of endurance trained cyclists. Int J Sports Med. 2004 May;25(4):247-51.

13. Jung A, Staus P, Lindner HJ, Schuppe HC. Influence of moderate cycling on scrotal temperature. Int J Androl. 2008 Aug;31(4):403-7.

14. Tartibian B, Maleki, BH. The effects of honey supplementation on seminal plasma cytokines, oxidative stress biomarkers, and antioxidants during 8 weeks of intensive cycling training. J Androl. 2012 MayJun;33(3):449-61. doi: 10.2164/jandrol.110.012815.

15. Maleki BH, Tartibian B, Vaamonde D. The effects of 16 weeks of intensive cycling training on seminal oxidants and antioxidants in male road cyclists. Clin J Sport Med. 2014 Jul;24(4):302-7. doi: 10.1097/ JSM.0000000000000051.

16. Meeker JD, Godfrey-Bailey L, Hauser R. Relationships between serum hormone levels and semen quality among men from an infertility clinic. J Androl. 2007 May-Jun;28(3):397-406.

17. Vaamonde D, Da Silva-Grigoletto ME, García-Manso JM, Vaamonde-Lemos R, Swanson RJ, Oehninger SC. Response of semen parameters to three training modalities. Fertil Steril. 2009 Dec;92(6):1941-6. doi: 10.1016/j.fertnstert.2008.09.010 